doi: $10.2306 /$ scienceasia1513-1874.2013.39S.006

\title{
Analysis of the Wenchuan aftershock data
}

\author{
Hai-Yen Siew ${ }^{\mathrm{a}, *}$, Ah-Hin Pooi ${ }^{\mathrm{b}}$ \\ a Meiji Institute for Advanced Study of Mathematical Sciences, Meiji University, 4-21-1 Nakano, Nakano-ku, \\ Tokyo 164-8525, Japan \\ b Sunway University Business School, Sunway University, No.5 Jalan Universiti, Bandar Sunway, \\ 46150 Petaling Jaya, Malaysia
}

*Corresponding author, e-mail: siew@meiji.ac.jp

Received 7 Jan 2013

Accepted 5 Apr 2013

\begin{abstract}
We analyse the aftershocks of the Wenchuan earthquake in 2008 using a multivariate non-normal distribution fitted to the observations for the variables selected from the inter-aftershock times, depths, magnitudes, sines and cosines of the azimuths, slant angles, and slip angles of a number $l+1$ of consecutive aftershocks. From the multivariate distribution, we find a prediction interval for the occurrence time of the next aftershock when the values of the other selected variables are given. The performance of the prediction interval is assessed by its ability to cover the observed occurrence time of the next aftershock, and by the average length of the interval. A similar prediction interval is also obtained for the magnitude of the next aftershock. It is found that as $l$ increases from $1-4$, while the performance of the prediction interval for the magnitude of the aftershock improves only slightly, that of the prediction interval for the inter-aftershock time improves quite substantially. The Wenchuan model with $l=4$ is also used for predicting the inter-aftershock time in the 2011 Tohoku earthquake. It is found that the drop in the coverage probability of the prediction interval for the next aftershock time in the Tohoku earthquake is only about $8 \%$, indicating that the characteristics of the aftershocks at different locations of the earth might have some similarities.
\end{abstract}

KEYWORDS: Tohoku, earthquake, inter-aftershock time, magnitude, prediction intervals

\section{INTRODUCTION}

An aftershock is a smaller earthquake that occurs after a previous large earthquake, in the same region of the main shock. Earthquakes and aftershocks follow several empirical laws. The Omori law proposed by Omori in 1894 describes the aftershock frequency as one which decreases by roughly the reciprocal of time after the main shock. Bath's law states that the differences in magnitude between a mainshock and its largest aftershock is constant, regardless of the mainshock magnitude. Gutenberg-Richter's law states that in any given region and time period, the number $N$ of earthquakes having a magnitude of at least $M$ is given by $N=10^{(a-b M)}$, where $a$ and $b$ are constants. While tending to follow these laws, the actual times of occurrence and magnitudes of the earthquakes and aftershocks are stochastic. Ref. 1 used a point process technique to study the temporal component of the earthquake activity. More sophisticated models which express the conditional intensity function of aftershocks as a function of the occurrence times and magnitudes of the previous aftershocks can be found in Refs. 2,3. Some authors included the spatial location of the shocks in their models ${ }^{4,5}$.
Presently, we fit a multivariate non-normal distribution called the multivariate power-normal distribution to the variables given by the inter-aftershock times, depths, magnitudes, sines and cosines of the azimuths, slant angles, slip angles of the current aftershock and $l-1$ previous shocks, and the occurrence time of the next aftershock. From the multivariate distribution, we find the prediction interval for the occurrence time of the next aftershock when the values of the after variables are given. When $l$ varies from $1-$ 4 , the prediction interval is found to have a coverage probability which is close to the target value, and there is a substantial decrease in the average length of the prediction interval. The omission of the sines and cosines of the three angles is found to have an obvious effect on the average length of the prediction interval when $l \leqslant 3$.

A similar prediction interval is also obtained for the magnitude of the next aftershock. When $l$ varies from $1-4$, the prediction interval is found to have a coverage probability which is close to the target value, and there is only a slight decrease in the average length of the prediction interval. The omission of the sines and cosines of the three angles is found to result in a slight increase in the average length of the prediction 
interval.

The layout of the paper is as follows. Next we define the variables which will be included to form the multivariate power-normal distribution. Then we describe the method given in Ref. 5 for deriving the conditional probability density function (pdf) of a particular variable from the multivariate power-normal distribution. The method given in Ref. 5 for fitting the multivariate power-normal distribution is described and finally we give the coverage probabilities and average lengths of the prediction intervals for the occurrence time and magnitude of the next aftershock in the Wenchuan earthquake.

\section{VARIABLES INCLUDED FOR FORMING MULTIVARIATE POWER-NORMAL DISTRIBUTION}

Let $V_{i}$ be the time of occurrence of the $i$ th aftershock of the earthquake which occurred at time $V_{0}$. Next, let $\boldsymbol{h}_{i}=\left(t_{i}, d_{i}, m_{i}, S_{i}^{(1)}, C_{i}^{(1)}, S_{i}^{(2)}, C_{i}^{(2)}, S_{i}^{(3)}, C_{i}^{(3)}\right)$ be the vector of characteristics of the $i$ th aftershock. The components in $\boldsymbol{h}_{i}$ are defined as: $t_{i}=V_{i}-V_{i-1}$ is the inter-aftershock time, $d_{i}$ the depth, $m_{i}$ the magnitude, $S_{i}^{(j)}$ and $C_{i}^{(j)}, j=1,2,3$, respectively, the sinus and cosines of the azimuths, slant angles, and slip angles, of the $i$ th aftershock.

In this paper, we try to predict the time $t_{i+1}$ elapsed before the occurrence of the $(i+1)$ th aftershock using the present value $\boldsymbol{h}_{i}$ and the lag values $\boldsymbol{h}_{i-1}, \ldots, \boldsymbol{h}_{i-l+1}$, where $l=1,2, \ldots$ To carry out the prediction, we first find a multivariate powernormal distribution for $\left(\boldsymbol{h}_{i-l+1}, \ldots, \boldsymbol{h}_{i-1}, \boldsymbol{h}_{i}, t_{i+1}\right)$, which has $9 l+1$ components. From the multivariate power-normal distribution, we find the conditional distribution for $t_{i+1}$ when the values of $\boldsymbol{h}_{i}, \boldsymbol{h}_{i-1}, \ldots, \boldsymbol{h}_{i-l+1}$ are given. The interval formed by the $100(\alpha / 2) \%$ and $100(1-\alpha / 2) \%$ points of the conditional distribution may then be regarded as a prediction interval for the future value $t_{i+1}$. When the above $100(\alpha / 2) \%$ point is negative, we may set the lower limit of the prediction interval to be zero. The performance of the prediction interval may be assessed by its ability to cover the future value $t_{i+1}$, and its average length.

\section{CONDITIONAL PDF DERIVED FROM MULTIVARIATE POWER-NORMAL DISTRIBUTION}

Ref. 6 considered the following power transformation

$$
\begin{aligned}
\tilde{\epsilon}= & \left(\lambda^{+}, \lambda^{-}, z\right) \\
& = \begin{cases}{\left[(1+z)^{\lambda^{+}}-1\right] / \lambda^{+},} & z \geqslant 0, \lambda^{+} \neq 0, \\
\log (1+z), & z \geqslant 0, \lambda^{+}=0, \\
-\left[(1-z)^{\lambda^{-}}-1\right] / \lambda^{-}, & z<0, \lambda^{-} \neq 0, \\
-\log (1-z), & z<0, \lambda^{-}=0 .\end{cases}
\end{aligned}
$$

If $z$ has the standard normal distribution, then $\tilde{\epsilon}$ has a non-normal distribution which is derived by a type of power transformation of a random variable with normal distribution. We may say that $\tilde{\epsilon}$ has a powernormal distribution.

We may now use the univariate power-normal distribution to obtain the multivariate power-normal distribution. First let $\boldsymbol{y}$ be a vector consisting of $k$ correlated random variables. The vector $y$ is said to have a $k$-dimensional power-normal distribution with parameters $\boldsymbol{\mu}, \boldsymbol{H}, \lambda_{i}^{+}, \lambda_{i}^{-}, \sigma_{i}, 1 \leqslant i \leqslant k$, if

$$
\boldsymbol{y}=\boldsymbol{\mu}+\boldsymbol{H} \epsilon
$$

where $\boldsymbol{\mu}=E(\boldsymbol{y}), \boldsymbol{H}$ is an orthogonal matrix, $\epsilon_{1}, \ldots, \epsilon_{k}$ are uncorrelated, for

$$
\epsilon_{i}=\frac{\sigma_{i}\left[\tilde{\epsilon}_{i}-E\left(\tilde{\epsilon}_{i}\right)\right]}{\sqrt{\operatorname{Var}\left(\tilde{\epsilon}_{i}\right)}}
$$

where $\sigma_{i}>0$ is a constant, and $\tilde{\epsilon}_{i}$ has a power-normal distribution with parameters $\lambda_{i}^{+}$and $\lambda_{i}^{-}$. When the values of $y_{1}, \ldots, y_{k-1}$ are given, we may find an approximation for the conditional pdf of $y_{k}$ by using the following numerical procedure.

(1) Select a large integer $N_{p}>0$ and compute $y_{k}^{\left(i_{p}\right)}=y_{k}^{-}+\left(i_{p}-1\right) h, 1 \leqslant i_{p} \leqslant N_{p}$, where $y_{k}^{+}$and $y_{k}^{-}$are such that $P\left(y_{k}^{-}<y_{k} \leqslant y_{k}^{+}\right)$is close to 1 , and $h=\left(y_{k}^{+}-y_{k}^{+}\right) / N_{p}$.

(2) Form the vector $\boldsymbol{y}^{\left(i_{p}\right)}=\left(y_{1}, \ldots, y_{k-1}, y_{k}^{\left(i_{p}\right)}\right)^{\prime}$ and find the value of $\boldsymbol{\epsilon}^{\left(i_{p}\right)}$ such that

$$
\boldsymbol{y}^{\left(i_{p}\right)}=\boldsymbol{\mu}+\boldsymbol{H} \boldsymbol{\epsilon}^{\left(i_{p}\right)} .
$$

(3) Replace $\left(\lambda^{+}, \lambda^{-}\right)$in (1) by $\left(\lambda_{i}^{+}, \lambda_{i}^{-}\right)$and find $z$ such that $\epsilon_{i}=\epsilon_{i}^{\left(i_{p}\right)}$. Let the answer of $z$ be denoted by $z_{i}^{\left(i_{p}\right)}$. 
(4) Compute

$$
f_{i_{p}}=\prod_{i=1}^{k} \frac{\exp \left[-\left(z_{i}^{\left(i_{p}\right)}\right)^{2} / 2\right]}{\sqrt{2 \pi}\left|\left(\mathrm{d} \epsilon_{i} / \mathrm{d} z_{i}\right)_{z_{i}=z_{i}^{\left(i_{p}\right)}}\right|} .
$$

(5) Estimate the conditional pdf (evaluated at $y_{k}^{\left(i_{p}\right)}$ ) of $y_{k}$ by $f_{i_{p}} / \sum_{i_{p}=1}^{N_{p}} f_{i_{p}}$..

\section{ESTIMATION OF PARAMETERS IN MULTIVARIATE POWER-NORMAL DISTRIBUTION}

We may use the following procedure to estimate the parameters in the multivariate power-normal distribution for a vector of which the $i$ th observed value is $\left(\boldsymbol{h}_{i-l+1}, \ldots, \boldsymbol{h}_{i-1}, \boldsymbol{h}_{i}, t_{i+1}\right)$.

(1) Let $L=9 l$ and

$$
\tilde{\boldsymbol{r}}^{\left(n_{1}\right)}=\left(\boldsymbol{h}_{i-l+1}, \ldots, \boldsymbol{h}_{i-1}, \boldsymbol{h}_{i}, t_{i+1}\right)^{\prime}
$$

when $i=n_{1}$. The value $\tilde{\boldsymbol{r}}^{\left(n_{1}\right)}$ may be viewed as the $n_{1}$ th observed value of a certain $(L+1) \times 1$ vector $\tilde{\boldsymbol{r}}$ of random variables.

(2) Compute

$$
\bar{r}_{i}=\frac{1}{N_{1}} \sum_{n_{1}=1}^{N_{1}} \tilde{r}_{i}^{\left(n_{1}\right)}
$$

and

$$
\begin{aligned}
& m_{i j}^{\left(k_{1}, k 2\right)} \\
& =\frac{1}{N_{1}} \sum_{n_{1}=1}^{N_{1}}\left(\tilde{r}_{i}^{\left(n_{1}\right)}-\bar{r}_{i}\right)^{k_{1}}\left(\tilde{r}_{j}^{\left(n_{1}\right)}-\bar{r}_{j}\right)^{k_{2}},
\end{aligned}
$$$$
1 \leqslant i, j \leqslant L+1 \text {. }
$$

(3) Compute the $L+1$ eigenvectors of the variancecovariance matrix $\left\{m_{i j}^{(1,1)}-m_{i j}^{(1,0)} m_{i j}^{(0,1)}\right\}$ and form the matrix $\boldsymbol{H}_{1}$ of which the $i$ th column is the $i$ th eigenvector.

(4) Compute $\boldsymbol{s}^{\left(n_{1}\right)}=\boldsymbol{H}_{1}^{\prime}\left(\tilde{\boldsymbol{r}}^{\left(n_{1}\right)}-\overline{\boldsymbol{r}}\right)$.

(5) Compute

$$
m_{i}^{(k)}=\frac{1}{N_{1}} \sum_{n_{1}=1}^{N_{1}}\left(s_{i}^{\left(n_{1}\right)}\right)^{k},
$$

$1 \leqslant i \leqslant L+1, k=2,3,4$.

(6) Find $\left(\lambda_{i}^{+}, \lambda_{i}^{-}\right)$and $\sigma_{i}$ such that $E\left(\epsilon_{i}^{k}\right)=m_{i}^{(k)}$, where $\epsilon_{i}$ is defined in (2) and $1 \leqslant i \leqslant l+1$, $k=2,3,4$.
Table 1 Coverage probability and expected length of prediction interval for next aftershock time measured in days $(\alpha=0.05)$.

\begin{tabular}{lcccc}
\hline$l$ & \multicolumn{2}{c}{ Coverage Probability } & \multicolumn{2}{c}{ Expected Length } \\
\cline { 2 - 5 } & With angles & Without angles & With angles & Without angles \\
\hline 1 & 0.9512 & 0.9561 & 1.5589 & 1.7736 \\
2 & 0.9314 & 0.9412 & 1.1231 & 1.1547 \\
3 & 0.9360 & 0.9409 & 1.0944 & 1.1951 \\
4 & 0.9303 & 0.9208 & 0.9303 & 0.9322 \\
\hline
\end{tabular}

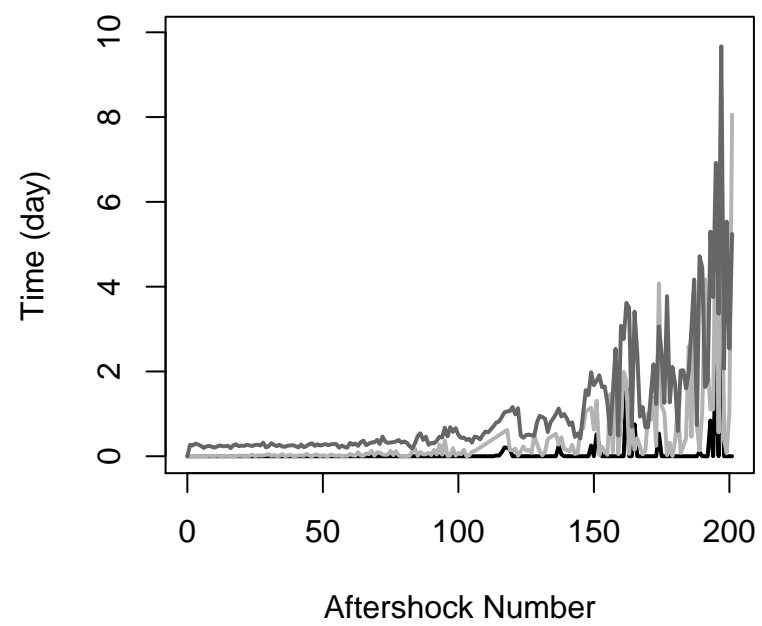

Fig. 1 Prediction intervals for next aftershock time in the 2008 Wenchuan earthquake: upper limit (dark grey), observed time (light grey), lower limit (black).

(7) The variable $\tilde{\boldsymbol{r}}$ may then be expressed as $\tilde{\boldsymbol{r}}=$ $\overline{\boldsymbol{r}}+\boldsymbol{H}_{1} \boldsymbol{\epsilon}$ which has an $(L+1)$-dimensional multivariate power-normal distribution with parameters

$\overline{\boldsymbol{r}}, \boldsymbol{H}_{1}, \lambda_{i}^{+}, \lambda_{i}^{-}, \sigma_{i}, i=1, \ldots, L+1$.

\section{PREDICTION OF NEXT AFTERSHOCK TIME AND MAGNITUDE OF WENCHUAN EARTHQUAKE}

The Wenchuan aftershocks from 12 May 2008 to 7 August 2008 with magnitude larger than $M_{W} 3.0$ shall be analysed in this section. By using the results in previous sections, we obtain the coverage probabilities and expected lengths of the prediction intervals for the occurrence time and magnitude of the next aftershock. The numerical results thus obtained are shown in Table 1 and Table 2.

Table 1 shows that as $l$ increases from $1-4$, the prediction interval for the next aftershock time has a coverage probability which is not very far from the target value 0.95 , and there is a substantial decrease in the average length of the prediction interval irre- 
Table 2 Coverage probability and expected length of prediction interval for next aftershock magnitude measured in $M_{W}(\alpha=0.05)$.

\begin{tabular}{lcccc}
\hline$l$ & \multicolumn{2}{c}{ Coverage Probability } & \multicolumn{2}{c}{ Expected Length } \\
\cline { 2 - 5 } & With angles & Without angles & With angles & Without angles \\
\hline 1 & 0.9265 & 0.9415 & 1.4384 & 1.4888 \\
2 & 0.9559 & 0.9461 & 1.4212 & 1.4632 \\
3 & 0.9507 & 0.9405 & 1.4093 & 1.4658 \\
4 & 0.9455 & 0.9406 & 1.3984 & 1.4660 \\
\hline
\end{tabular}

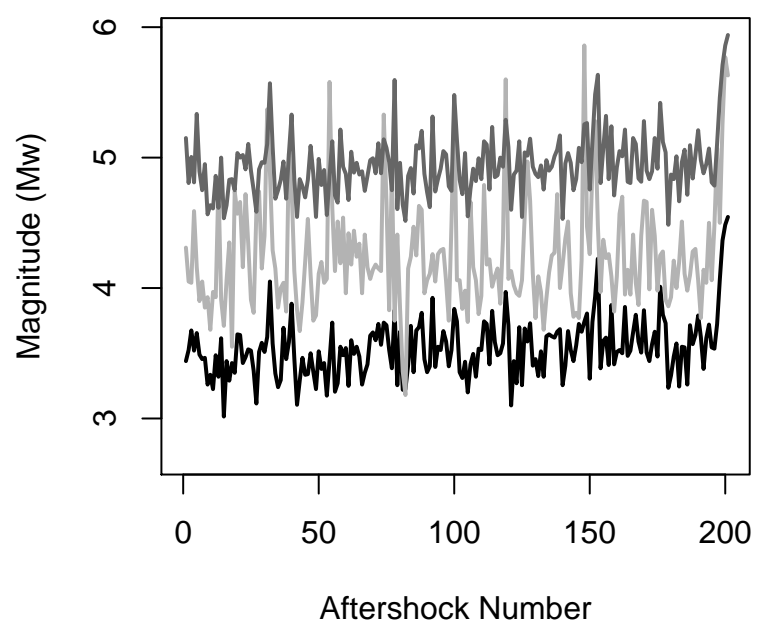

Fig. 2 Prediction intervals for the next aftershock magnitude in the 2008 Wenchuan earthquake: upper limit (dark grey), observed magnitude (light grey), lower limit (black).

spective of whether the sines and cosines of the three angles are included in the list of variables for forming the multivariate power-normal distribution. The table also shows that the omission of the angles has an obvious effect on the average length of the prediction interval when $l \leqslant 3$. The prediction intervals for $l=4$, with angles, are also shown in Fig. 1 .

From Table 2, we see that as $l$ increases from $1-4$, the prediction interval for the next aftershock magnitude has a coverage probability which is quite close to the target value 0.95 , and there is only a slight decrease in the average length of the prediction interval. The omission of the angles is found to yield a slight increase in the average length of the prediction interval. The prediction intervals for $l=4$, with angles, are given in Fig. 2.

When $l=4$, the Wenchuan model is next used to find prediction intervals for the next aftershock times in the 2011 Tohoku earthquake. The prediction intervals thus obtained are found to have a coverage probability of 0.8734 and an average length of 0.8867 day. These prediction intervals are displayed

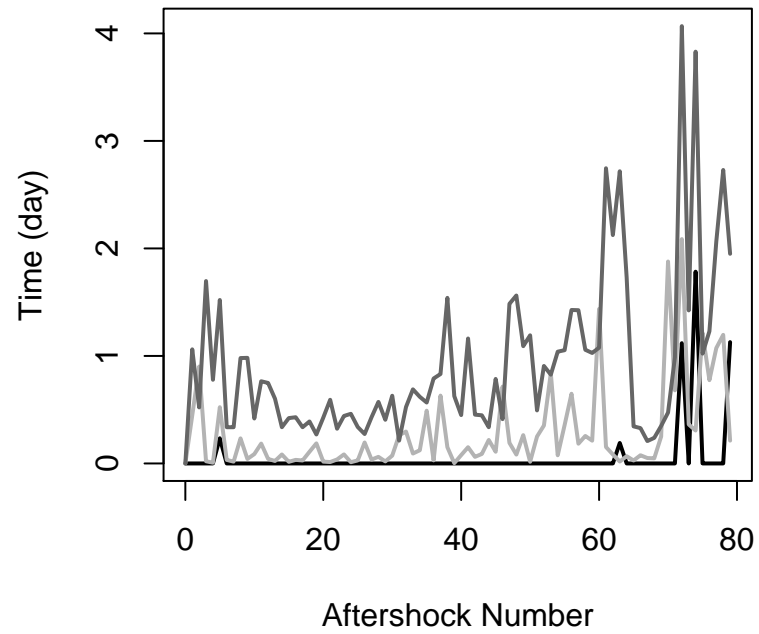

Fig. 3 Prediction intervals based on the Wenchuan model for next aftershock time in the 2011 Tohoku earthquake: upper limit (dark grey), observed time (light grey), lower limit (black).

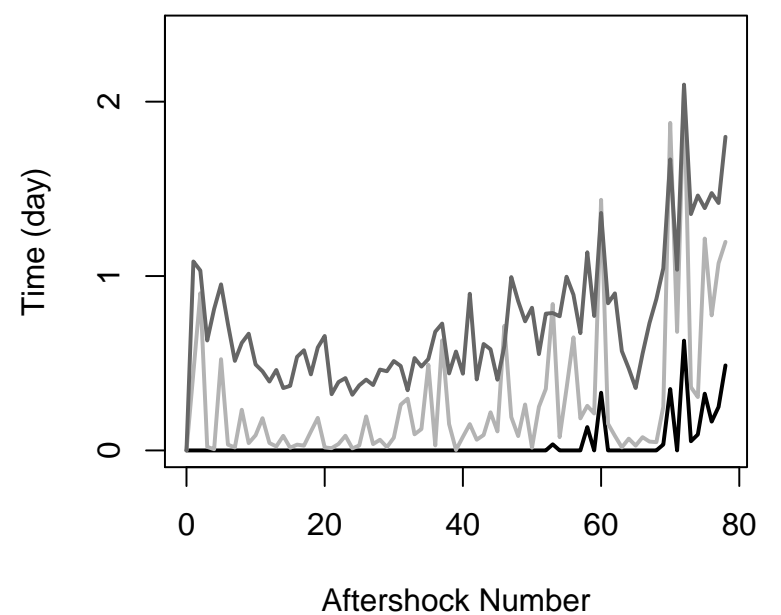

Fig. 4 Prediction intervals based on the Tohoku model for next aftershock time in the 2011 Tohoku earthquake: upper limit (dark grey), observed time (light grey), lower limit (black).

in Fig. 3. A multivariate power-normal distribution has also been obtained fitted to the 2011 Tohoku data. The corresponding prediction interval for the next aftershock times in the 2011 Tohoku earthquake are found to have a coverage probability of 0.9487 and an average length of 0.6974 day. These prediction intervals are shown in Fig. 4. Thus the prediction intervals based on the Wenchuan model for the next aftershock time in the 2011 Tohoku earthquake have poorer performance in terms of coverage probability 
and average length. However, the drop of only about $8 \%$ in coverage probability indicates that the characteristics of the aftershocks in different locations of the earth might have some similarities.

\section{CONCLUSIONS}

Aftershocks are dangerous because they are difficult to predict, can be of large magnitude, and can last for years. The present paper shows that the characteristics of the present and past aftershocks can help to construct shorter prediction intervals for the occurrence time and magnitude of the next aftershock. As the dataset analysed contains information only on aftershocks with magnitude larger than $M_{W} 3.0$, it would be useful to find out whether the inclusion of smaller magnitude aftershocks would help to further shorten the prediction intervals.

Acknowledgements: The Global COE program of Meiji University has provided funding to the first author for the present research. The financial support is gratefully acknowledged.

\section{REFERENCES}

1. Utsu T (1961) A statistical study on the occurrence of aftershocks. Geophys Mag 30, 521-605.

2. Ogata Y (1988) Statistical models for earthquake occurrences and residual analysis for point processes. J Amer Statist Assoc 83, 9-27.

3. Ogata Y (1989) Statistical model for standard seismicity and detection of anomalies by residual analysis. Tectonophysics 169, 159-74.

4. Ogata Y, Katsura K (1988) Likelihood analysis of spatial inhomogeneity for marked point patterns. Ann Inst Statist Math 40, 29-39.

5. Pooi AH (2012) A model for time series analysis. Appl Math Sci 6, 5735-48.

6. Yeo IK, Johnson RA (2000) A new family of power transformations to improve normality or symmetry. Biometrika 87, 954-9. 